\title{
Gap-junction uncoupling as a pharmacological strategy to prevent hypothermia-induced ventricular fibrillation
}

\author{
Erik S Dietrichs ${ }^{1,2,3}$, Karen Mcglynn ${ }^{4}$, Andrew Allan $^{4}$, Adam Connolly ${ }^{5}$, Martin Bishop ${ }^{5}$, \\ Francis Burton ${ }^{4}$, Rachel Myles ${ }^{4}$, Torkjel Tveita ${ }^{2}$, Godfrey L Smith ${ }^{4}$ \\ ${ }^{I}$ Clinical Pharmacology, University Hospital of Northern Norway, Norway, ${ }^{2}$ Anesthesia and Critical Care Research \\ Group, Department of Clinical Medicine, UiT, The Arctic University of Norway, Norway, ${ }^{3}$ Department of Research and \\ Education, Norwegian Air Ambulance Foundation, Norway, ${ }^{4}$ Institute of Cardiovascular \& Medical Sciences, \\ University of Glasgow, UK, ${ }^{5}$ Department of Biomedical Engineering, Division of Imaging Sciences \& Biomedical \\ Engineering, Kings College London, UK
}

Background: Ventricular arrhythmias and cardiac arrest emerge as the most important complication when rewarming victims of accidental hypothermia. Pharmacological treatment and prevention of such arrhythmias remains challenging and the underlying mechanisms are unclear. This randomized, experimental study assessed cardiac electrophysiology to find potential pharmacological strategies at temperatures occurring in therapeutic and accidental hypothermia.

Methods: Rabbit hearts were cooled to moderate (31C) and severe (17C) hypothermia to investigate electrophysiological mechanisms underlying hypothermia-induced increased risk for ventricular fibrillation. Panoramic optical mapping, electrode-based conduction velocity measurements and ventricular fibrillation threshold was investigated. A computational model was used to confirm potential target mechanisms, prior to testing pharmacological interventions in vitro.

Results: Ventricular conduction was insensitive to cooling at $31 \mathrm{C}$, but slowed at $17 \mathrm{C}$. Ventricular activation was unaffected at $31 \mathrm{C}$ whilst repolarisation was significantly prolonged. At $17 \mathrm{C}$ there were proportionally similar delays in both activation and repolarisation. These changes were reflected in the QRS and QT intervals of ECG recordings. Ventricular fibrillation threshold was significantly reduced at $31 \mathrm{C}$, but at $17 \mathrm{C}$ ventricular fibrillation threshold was $>2 \mathrm{x}$ baseline $(37 \mathrm{C})$. At $31 \mathrm{C}$, transverse conduction was relatively insensitive to cooling versus longitudinal conduction, but at 17C both transverse and longitudinal conduction were proportionately reduced to a similar extent. Pharmacological intervention with the gap junction uncoupler heptanol had a larger relative effect on transverse than longitudinal conduction, and was at $31 \mathrm{C}$ able to restore the transverse/longitudinal conduction ratio, returning ventricular fibrillation threshold to baseline values.

Conclusions: Moderate hypothermia does not significantly change ventricular conduction time, but creates repolarisation abnormalities and is pro-arrhythmic. Risk for ventricular fibrillation appear to be linked to a lower temperature sensitivity of gap junctions. Pharmacological intervention with the gap-junction uncoupler heptanol elevated ventricular fibrillation threshold to normothermic values and proved to be an effective anti-arrhythmic intervention at 31C. 